\title{
A PROCEDURE FOR GENERATING IN-CABINET RESPONSE SPECTRA BASED ON STATE-SPACE MODEL IDENTIFICATION BY IMPACT TESTING
}

\author{
SUNG GOOK CHO ${ }^{1}$, JINTAO CUI ${ }^{2}$, and DOOKIE KIM ${ }^{2, *}$ \\ ${ }^{1}$ JACE KOREA R\&D Center \\ Sangdeawon-dong 190-1, Jungwon-gu, Seongnam-si, Gyeonggi, 462-120, Korea \\ ${ }^{2}$ Department of Civil and Environmental Engineering, Kunsan National University \\ Miryong, Kunsan, Jeonbuk, 573-701, Republic of Korea \\ *Corresponding author. E-mail : kim2kie@chol.com
}

Received August 17, 2010

Accepted for Publication April 29, 2011

The in-cabinet response spectrum is used to define the input motion in the seismic qualification of instruments and devices mounted inside an electrical cabinet. This paper presents a procedure for generating the in-cabinet response spectrum for electrical equipment based on in-situ testing by an impact hammer. The proposed procedure includes an algorithm to build the relationship between the impact forces and the measured acceleration responses of cabinet structures by estimating the state-space model. This model is used to predict seismic responses to the equivalent earthquake forces. Three types of structural model are analyzed for numerical verification of the proposed method. A comparison of predicted and simulated response spectra shows good convergence, demonstrating the potential of the proposed method to predict the response spectra for real cabinet structures using vibration tests. The presented procedure eliminates the uncertainty associated with constructing an analytical model of the electrical cabinet, which has complex mass distribution and stiffness.

KEYWORDS : In-cabinet Response Spectrum, Electrical Cabinet, In-situ Test, Impact Hammer, State-space Model, Seismic Response

\section{INTRODUCTION}

Active safety-related equipment is typically seismically qualified before installation within nuclear power plants. Seismic qualification (SQ) of equipment may be required not only when the equipment is installed in the plant but also when the state of the equipment is changed during operation. The most common SQ methods employ analysis, testing, a combination of testing and analysis, and/or experience data [1]. However, the experience data method is impractical in Korea due to a lack of such data from earthquake experience, and qualification tests also have certain drawbacks as they are typically performed in testing laboratories located away from the plant.

In addition, some types of equipment cannot be qualified by analysis or testing alone due to their size or complexity. It may also be impractical to test large equipment at full levels due to limitations in the vibration test equipment according to the circumstances. Furthermore, the commonly used shaking table test cannot be used on equipment already installed in operating plants because such equipment cannot be moved from its original position to mount on a test facility. In contrast to the above methods, in-situ vibration tests like the impact test can provide an efficient, effective way to determine the modal properties of those cabinets instead of using a large shaking table system. A combined method of testing and analysis can be used as an aid to the SQ of large and complex systems per IEEE-344 [1]. Equipment vibration tests are performed not only for SQ but also for measuring modal properties and for validating the analytical model.

The SQ of the device or the relay mounted on or within the cabinet is performed by testing it to a level equal to or greater than the established response at the mounting location. The seismic input for the components inside the cabinet is defined in terms of an in-cabinet response spectrum (ICRS) which can be calculated from the response at the mounting location of the cabinet. The ICRS can be generated by seismic analyses or shaking table tests of the entire cabinet. As mentioned above, a full-scale shaking table test of the operating equipment is impractical. It is possible to generate a realistic ICRS by 
seismic analysis after obtaining the accurate modal parameters of the cabinet. In-situ vibration tests can be performed on an electrical cabinet in the field to obtain its modal properties from which the ICRS can be calculated. This is a cost-effective alternative compared to de-energizing the equipment, disconnecting the cables, and moving the equipment to a laboratory for a shaking table test.

As part of industry efforts to generate the ICRS of equipment in older operating nuclear power plants, EPRI [2] developed generic seismic amplification factors for electrical cabinets that are valid for any location in a cabinet. These amplification factors are used to scale the floor response spectra (FRS) at the base of the cabinet to the amplified response spectra. However, the amplification factors in the EPRI report [2] are typically very conservative and tend to give unrealistic spectra. Gupta et al. [3] and Gupta and Yang [4] presented analytical approaches to evaluate the ICRS based on two-degree-of-freedom Ritz vectors. Yang and Gupta [5] also developed a computer program to generate an ICRS by using the formulations of the Rayleigh-Ritz method. The modified Ritz vector method for generating an ICRS was presented by Rustogi and Gupta [6] to consider the uplift of the cabinet base by horizontal rocking. These methods are based on analytical approaches. Therefore, for these methods the analytical models must be constructed first to represent the dynamic characteristics of the equipment.

However, it is not easy to model electrical equipment analytically. In order to avoid constructing the analytical model, Koo et al. [7] developed an effective method to predict the seismic response of a structure based on statespace model identification. The study by Koo et al. [7] focused on verification of the predicting method by the shaking table test for a simple specimen. Further studies are required to extend this study to predict the ICRS of the cabinet and to present further verifications for more complex models. This study developed a discrete-time state-space equation (SSE) to predict the equivalent seismic forces applied at the cabinet and a method to estimate the seismic responses of the cabinet by numerical integration of the equation of motion. This paper introduces the procedure to predict the ICRS and describes the numerical simulation tests for its verification.

\section{CRS PREDICTION}

\subsection{Prediction Procedure}

The seismic response of a structure can be estimated after its dynamic characteristics are reasonably identified. This study proposes a procedure to estimate the ICRS of electrical equipment based on an in-situ vibration test using an impact hammer. The proposed procedure eliminates the uncertainty associated with constructing an analytical model of an electrical cabinet that has a complex mass distribution and stiffness.

The ICRS prediction procedure used in this study is summarized in Fig. 1. This procedure starts from generating an artificial earthquake motion compatible with the target response spectrum. The artificial earthquake motion may be a time-history acceleration as shown in Fig. 2. The procedure also includes identification of the state-space equation by measuring the response and the impulse from impact tests. The seismic responses under generated artificial earthquakes are then predicted and converted to ICRS.

\subsection{Earthquake-equivalent Load}

The earthquake-equivalent load at the mass point of the cabinet as shown in Fig. 3 is expressed as:

$$
F(t)=-M\{1\} \ddot{u}_{g}
$$

where $\ddot{u}_{g}$ denotes the base acceleration as the earthquake input motion and $\{1\}$ is the column vector filled with ones. $M$ is the mass matrix of the cabinet structure.

The equation of motion for a linear dynamic system subjected to an earthquake-equivalent load can be expressed as:

$$
M \ddot{u}+C \ddot{u}+K u=F(t)
$$

where $M, C, K$ are the mass, damping and stiffness matrices of the cabinet structure, respectively, and $u$ denotes the displacement response vector to the effective load vector of $F(t)$.

From Eq. (2), the acceleration responses can be derived as follows:

$$
\ddot{u}=\frac{1}{M}(-C \dot{u}-K u+F(t))
$$

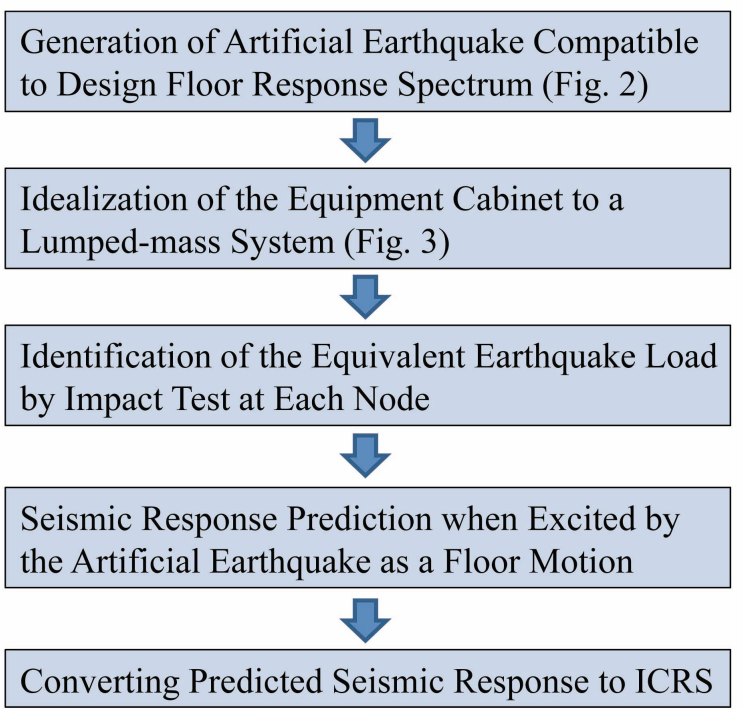

Fig. 1. ICRS Prediction Flowchart 
The relationship can be rewritten as follows [8]:

$$
\begin{gathered}
{\left[\begin{array}{c}
\dot{u} \\
\ddot{u}
\end{array}\right]=\left[\begin{array}{cc}
0 & I \\
-\frac{K}{M} & -\frac{C}{M}
\end{array}\right]\left[\begin{array}{l}
u \\
\dot{u}
\end{array}\right]+\left[\begin{array}{c}
0 \\
\frac{1}{M}
\end{array}\right] F(t)} \\
\ddot{u}=\left[\begin{array}{cc}
-\frac{K}{M} & -\frac{C}{M}
\end{array}\right]\left[\begin{array}{l}
u \\
\dot{u}
\end{array}\right]+\left[\frac{1}{M}\right] F(t)
\end{gathered}
$$

which is the form of the state space equation in continuoustime. Equations (4) and (5) can be reproduced by assigning $x(t)=\left[\begin{array}{c}\dot{\mathrm{u}} \\ \ddot{\mathrm{u}}\end{array}\right], y(t)=\ddot{\mathrm{u}}, u(t)=F(t), A=\left[\begin{array}{c}{ }^{0} \frac{K}{M}-\frac{I}{M} \\ -\frac{C}{M}\end{array}\right], B=\left[\begin{array}{c}0 \\ \frac{1}{M}\end{array}\right]$, and $D=\left[\frac{1}{M}\right]$ to be the representative form of the state space equation as follows:

$$
\begin{aligned}
& \dot{x}(t)=A x(t)+B u(t) \\
& y(t)=C x(t)+D u(t)
\end{aligned}
$$

where $x(t), y(t)$ and $u(t)$ are the state values, output acceleration response and input force at time $t$ in this study. To obtain the discrete-time state space equation, first consider the linear time-invariant homogeneous equation as follows:

$$
\dot{x}(t)=A x(t)
$$

The solution to the previous equation always exists and can be expressed as follows:

$$
x(t)=\Phi\left(t, t_{0}\right) x_{0}
$$

The transition matrix $\Phi\left(t, t_{0}\right)$ is the solution of the matrix differential equation as

$$
\dot{\Phi}\left(t, t_{0}\right)=A \Phi\left(t, t_{0}\right), \Phi\left(t_{0}, t_{0}\right)=I
$$

which has the explicit form

$$
\begin{aligned}
\Phi\left(t, t_{0}\right) & =\Phi\left(t-t_{0}\right)=e^{A\left(t-t_{0}\right)} \\
& =1+A\left(t-t_{0}\right)+\frac{1}{2 !}\left[A\left(t-t_{0}\right)\right]^{2}+\cdots
\end{aligned}
$$

This is the Taylor series expansion and it converges for all $A$.

Now, consider the state equation as follows:

$$
\dot{x}(t)=A x(t)+B u(t), \quad x\left(t_{0}\right)=x_{0}
$$

If $u(t)$ is piecewise continuous for all $t$, we have

$$
\begin{aligned}
& x(t)=\Phi\left(t-t_{0}\right) x_{0}+\int_{0}^{t} \Phi(t-\tau) B u(\tau) d \tau \quad, \quad t \geq t_{0} \\
& =e^{A\left(t-t_{0}\right)} x_{0}+\int_{0}^{t} e^{A(t-\tau)} B u(\tau) d \tau
\end{aligned}
$$

Thus, the discrete-time state space equation can be summarized as follows:

$$
\begin{aligned}
& x\left(t_{k+1}\right)=e^{A\left(t_{k+1}-t_{k}\right)} x\left(t_{k}\right)+\int_{t_{k}}^{t_{k+1}} e^{A\left(t_{k+1}-t_{k}\right)} B d \tau u\left(t_{k}\right) \\
& y\left(t_{k}\right)=C x\left(t_{k}\right)+D u\left(t_{k}\right)
\end{aligned}
$$
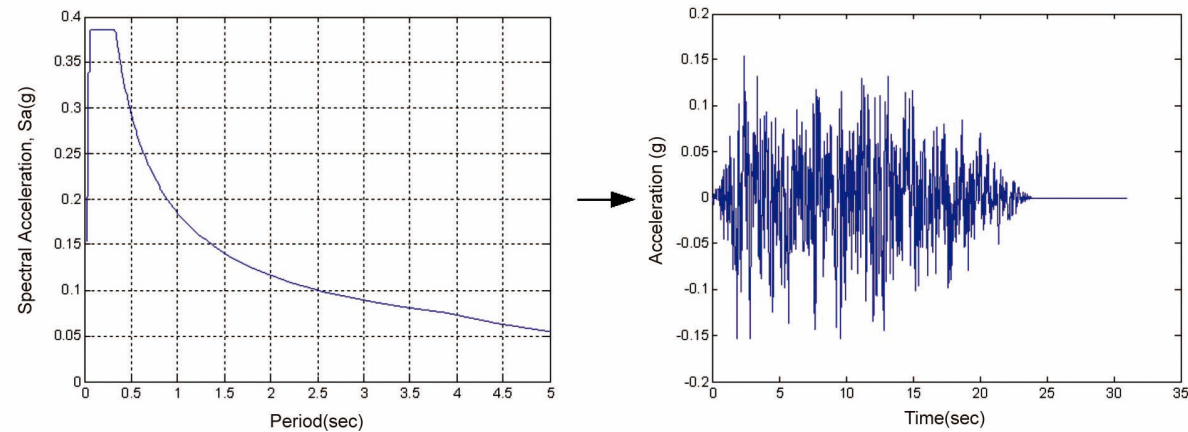

Fig. 2. Generation of an Artificial Earthquake Compatible with a Floor Response Spectrum
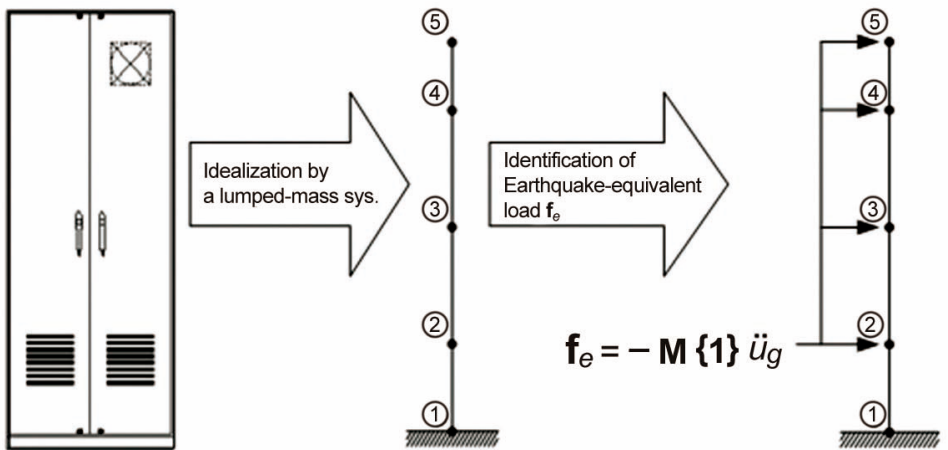

Fig. 3. Idealization of a Cabinet and Identification of its Earthquake-equivalent Load 
It can be written for the incremental time step as follows:

$$
\begin{aligned}
& x\left(t_{k+1}\right)=e^{A T} x\left(t_{k}\right)+\int_{0}^{T} e^{A T} B d \tau u\left(t_{k}\right) \\
& y\left(t_{k}\right)=C x\left(t_{k}\right)+D u\left(t_{k}\right)
\end{aligned}
$$

where $x\left(t_{k}\right), y\left(t_{k}\right)$ and $u\left(t_{k}\right)$ are the input force, the response acceleration and measurement noise vectors at time step $k(k=1,2, \ldots, n) ; n$ is the number of time steps, respectively. $A, B, C$ and $D$ are the system matrices.

\subsection{Stochastic Subspace Identification Method}

The relationship between the responses of the structure and the impact forces can be identified in the form of a discrete-time state space equation (SSE) as Eq. (14). Various algorithms have been developed for identification of the discrete-time SSE. This study uses the N4SID algorithm proposed by Overschee and Moor [9, 10], which is a variant of the subspace identification method and uses very simple parameters. N4SID has major advantages that are non-iterative and rapidly converged. The N4SID is derived by using geometric arguments and a systemic theoretical approach similar to the realization theory [11].

The state vector $x(k)$ is defined to be a linear combination of the past inputs and outputs [12]:

$$
x(k)=J p(k)
$$

where

$$
\begin{aligned}
p(k)=[ & y^{T}(k-1), \cdots y^{T}(k-N), u^{T}(k-1), \\
& \left.\cdots y^{T}(k-N), u^{T}(k-1), \cdots u^{T}(k-N)\right]
\end{aligned}
$$

and $p(k)$ is referred to as the "past" for sample $k$. The dimension of the past is the number of lags $N$. The state vector $x(k)$ is calculated from the data and is not specified to be a physical state of the system. After $J$ has been calculated (see below), the state vector can be estimated by Eq. (15). The state-space model matrices can then be estimated through linear regression.

$$
\left.\left[\begin{array}{ll}
\hat{A} & \hat{B} \\
\hat{C} & \hat{D}
\end{array}\right]=\operatorname{cov}\left(\left[\begin{array}{c}
x(k+1) \\
y(k)
\end{array}\right],\left[\begin{array}{l}
x(k) \\
u(k)
\end{array}\right]\right) \times \operatorname{cov}^{-1}\left(\begin{array}{l}
x(k) \\
y(k)
\end{array}\right],\left[\begin{array}{l}
x(k) \\
u(k)
\end{array}\right]\right)
$$

The calculation of $J$ distinguishes the various subspace algorithms from one another. In the N4SID approach, $J$ results from a series of geometric arguments (or linear algebraic arguments) based on a set of matrix equations for the evolution of a linear system [13] based on a weighted singular value decomposition (SVD) [14] as follows:

$$
\operatorname{svd}(\operatorname{cov}(p, \bar{f}))=\left[\begin{array}{ll}
U_{1} & U_{2}
\end{array}\right]\left[\begin{array}{cc}
S_{1} & 0 \\
0 & S_{2}
\end{array}\right]\left[\begin{array}{c}
V_{1}^{T} \\
V_{2}^{T}
\end{array}\right]
$$

where $f$ is the "conditional future" estimated by the past observations, $p(k), U_{1}, U_{2}, V_{1}$ and $V_{2}$ are orthonormal matrices $U U^{T}=U^{T} U=I$ and $V V^{T}=V^{T} V=I$, and $S_{1}$ is the singular value matrix with high contributions and $S_{2}$ is the singular value with negligibly small values on its diagonal.

N4SID only makes use of the lower dimensional submatrices with significant contributions by $U_{1}$ and $S_{1}$, and ignores tiny contributions by $U_{2}$ and $S_{2}$. By inspecting the whole singular value matrix, the size of $S_{1}$ can be determined as the number of significant or non-negligible singular values in the whole singular value matrix which is equal to the order of the SSE model.

\section{NUMERICAL VERIFICATION}

Numerical simulation tests for a given earthquake motion were performed on an electrical equipment cabinet to verify the algorithm of this study. The predicted seismic responses were obtained by applying the procedure proposed in this study. The simulated seismic responses are the analysis results computed by the numerical integration for the earthquake motions which are identical to the motions of the simulation tests.

In this section, three different analytical models are presented to be used in the numerical verification of the proposed algorithm, which are a lumped-mass beam stick model, a frame model, and a 3-dimensional finite element model to represent the cabinet structure, respectively. The models were constructed by considering the characteristics of a seismic monitoring system cabinet for nuclear pore plants whose main functions are alarming and monitoring seismic signals at the site of the plant. As shown in Figure 4, the cabinet includes very complex units and components inside. The electrical cabinet typically consists of an internal steel frame of channels and angles, thin steel plate covers enwrapping the frame, with intermediate diaphragms of thin steel plate inside. The cabinet has front and rear entrance doors and all structural components are interconnected with each other by bolting or spot welding.

Dynamic response analyses using the direct integration method were performed to obtain the vibration responses of the models due to the impact forces and the seismic motions. A 5\% damping factor as a percentage of critical damping was considered in the dynamic analyses. A general purpose structural analysis program [15] was used to generate the response acceleration signals in numerical simulation tests excited by an impact hammer and by seismic motions.

\subsection{Lumped-mass Beam Stick Model}

A typical instrument cabinet was selected as a model for verification of this study. For the first stage of verification, a lumped-mass beam stick model with four nodal points was constructed as shown schematically in Fig. 4. The lumped masses of an idealized system at each node were determined by referring to the mass distribution of an actual cabinet. The beam element was modeled using the equivalent properties with an area of $91.52 \times 10^{-4} \mathrm{~m}^{2}$ and a moment of inertia of $3.7934 \times 10^{-6} \mathrm{~m}^{4}$. The model properties 
were determined by referring to a previous study [16].

The impact hammer tests were performed at the four points from node 2 to node 5 continuously in time. $1 \mathrm{kN}$ of impact force was applied to each node and the acceleration responses were also collected at these nodes. Then the impact forces and the acceleration responses in the time domain (Figs. 5 and 6) were used as input-output data to build the state-space model. Practically, with more times of impacts at each node, a more reliable estimation
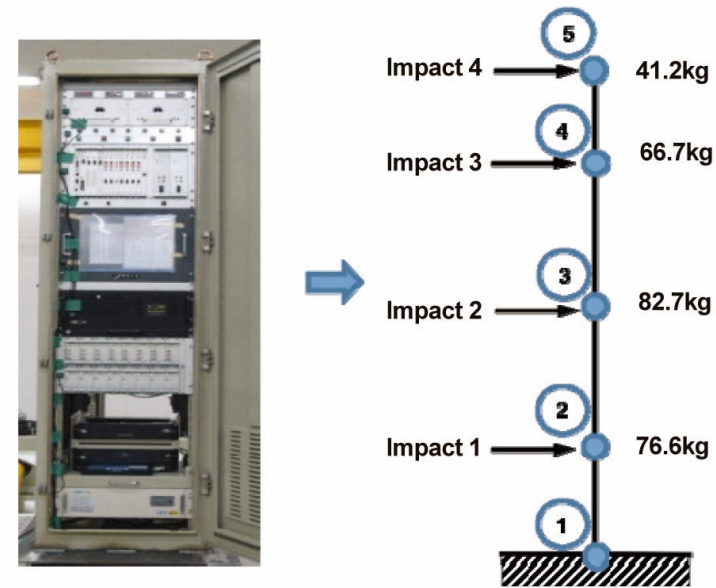

Fig. 4. Lumped-mass Beam Stick Model of the state space equation can be achieved. In this study, two times of impacts at each node were simulated to show the effectiveness and efficiency of the proposed procedure. Based on a set of input and output measurements, a statespace equation model was identified using N4SID. The order of the state-space equation was selected as 8 by inspecting the singular values obtained by Eq. (18).

The artificial earthquakes were generated as time history acceleration input motions at the cabinet base, which are compatible with a target response spectrum as shown in Fig. 2. For each of ten artificial earthquake cases, the equivalent earthquake force $f_{e}$ was calculated by feeding the measured base motion $\ddot{u}_{g}$ into the SSE model as an input to estimate earthquake responses. The simulated and predicted earthquake responses at nodes 3 and 4 are compared in Fig. 7. The predicted response is in reasonable agreement with the measured response as shown in Fig. 7.

The simulated and predicted seismic responses were then converted to ICRS to verify the effectiveness of the proposed procedure. The comparison of mean ICRS from ten sets of simulated and predicted seismic responses shows a good convergence as shown in Fig. 8.

\subsection{Frame Model}

The proposed algorithm was then verified through a detailed frame model as shown in Fig. 9. The beam elements were modeled using the equivalent properties with an area of $45.76 \times 10^{-4} \mathrm{~m}^{2}$ and a moment of inertia of
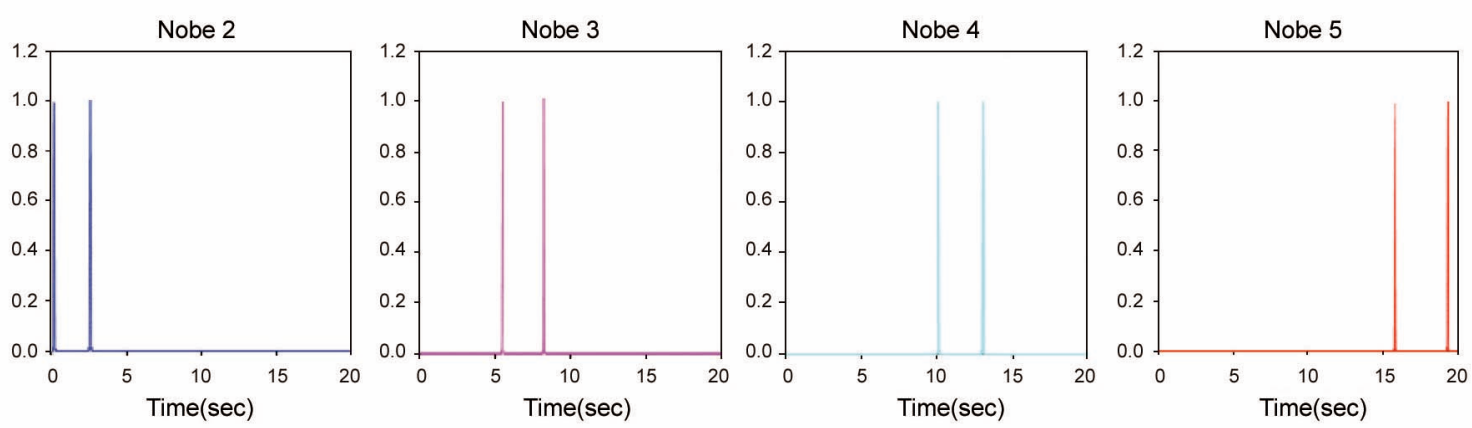

Fig. 5. Impact Forces for Lumped-mass Beam Stick Model
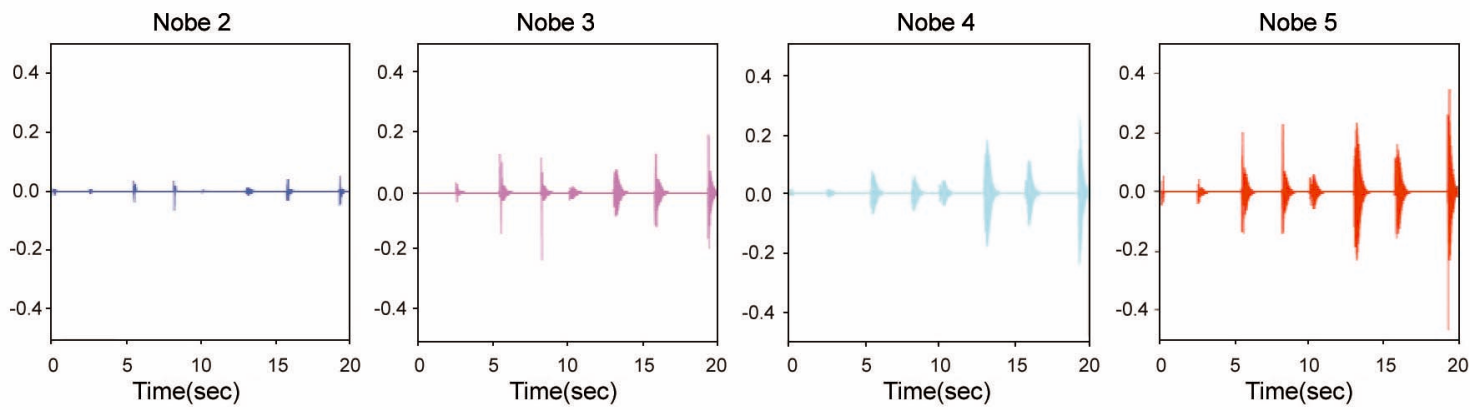

Fig. 6. Floor Acceleration Responses for the Lumped-mass Beam Stick Model 
$1.8967 \times 10^{-6} \mathrm{~m}^{4}$. To predict the seismic response based on the SSE model, the equivalent earthquake force $f_{e}$ was calculated by idealizing the model to a lumped-mass system.

The numerical simulation tests were performed in a manner similar to the lumped-mass beam stick model case. The impact hammering was applied at the four points from node 2 to node 5 on the frame model continuously
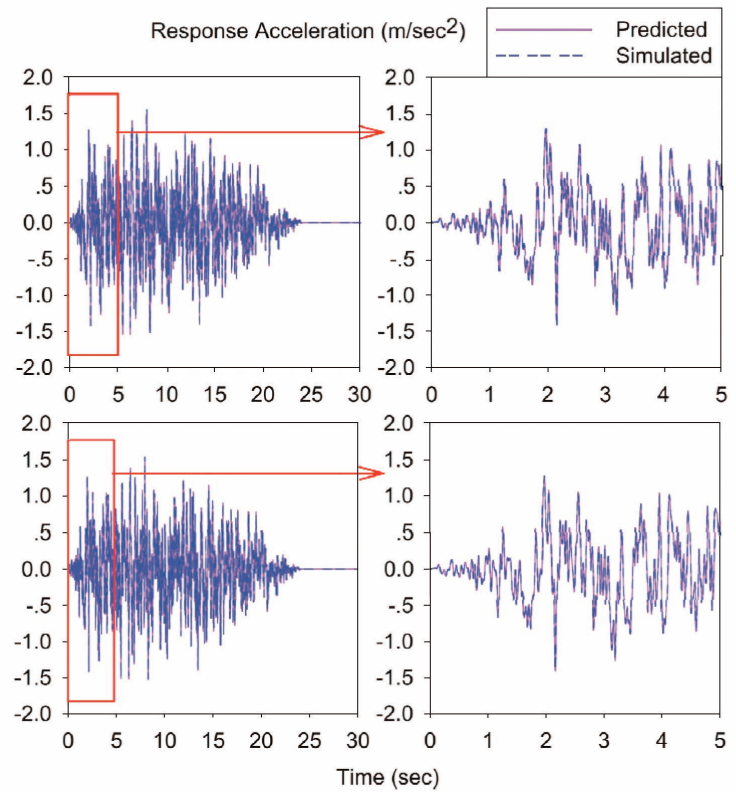

Fig. 7. Comparison of Seismic Acceleration Responses for the Lumped-mass Beam Stick Model
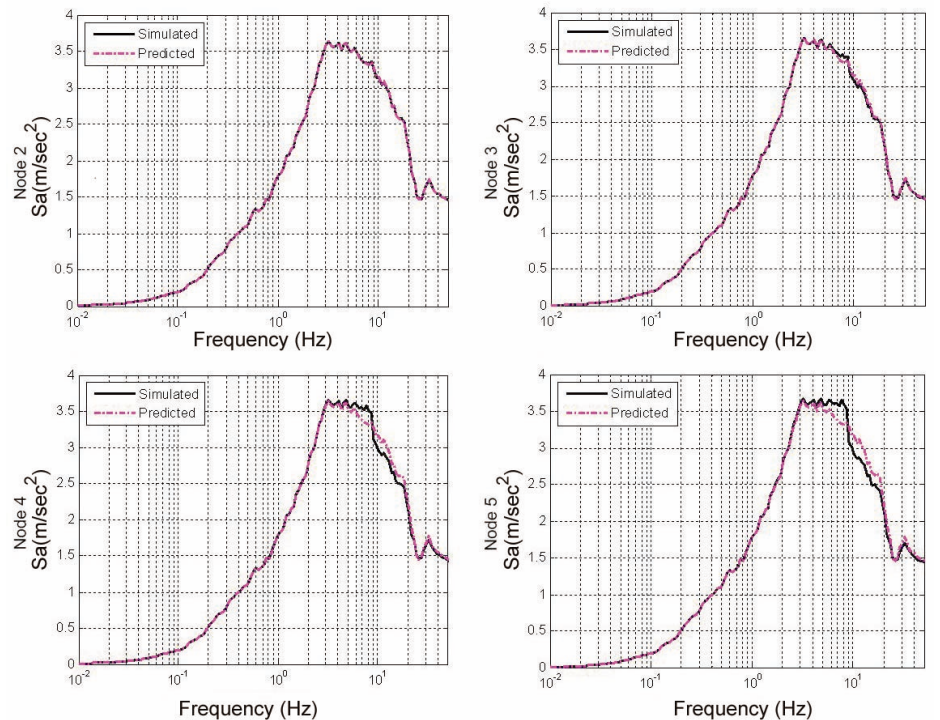

Fig. 8. Comparison of Simulated and Predicted ICRS's for the Lumped-mass Beam Stick Model in time, and the acceleration responses were also collected at these nodes. The impact forces and the acceleration responses in the time domain (Figs. 10 and 11) were used as input-output data to build the state-space model. A state-space equation model was identified based on a relationship between input and output signals.

The simulated and predicted earthquake responses at nodes 3 and 4 are compared in Fig. 12. The predicted response is in reasonable agreement with the measured response even with a little difference as shown in Fig. 12. The mean ICRS from ten sets of simulated seismic

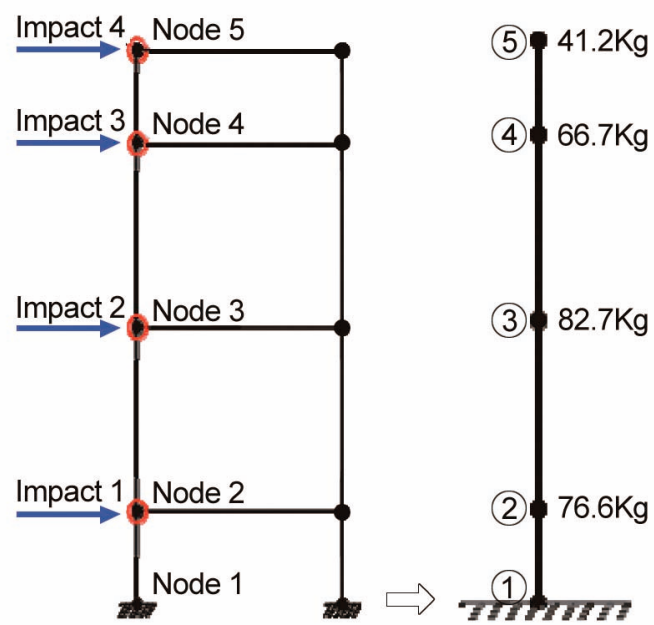

Fig. 9. Frame Model and Idealization of the Lumped-mass System 
responses shows good convergence with that obtained by prediction as shown in Fig. 13.

\subsection{3-Dimensional Finite Element Model}

The proposed algorithm was additionally verified through a detailed finite element model. In this verification, a 3-dimensional finite element model was constructed as shown in Fig. 14. This model was constructed by combining 3 -dimension plate elements and frame elements. The plate elements represent the roof and exterior wall plates, interior diaphragm plates, and floor plates. The frame elements were additionally modeled to represent the horizontal and vertical stiffeners in the interior of the cabinet. 108 frame elements and 203 plate elements were used to represent the stiffness of the model. The structural masses were automatically computed by inputting the mass density of the structural element. The additional masses for non-structural members and attachments like doors were inputted as lumped masses at the corresponding nodal points. This model was constructed by referring to a previous study [16].

The numerical simulation tests were performed and a state-space equation was identified in the same way as in the previous model cases. In order to identify the statespace equation model, the impact hammering was applied at the four nodes as shown in Fig. 14. The nodal masses of the 3-dimensional finite element model were calculated by considering the actual mass distribution of the cabinet. Fig. 15 and Fig. 16 show the impact forces and the acceleration responses in the time domain. The simulated
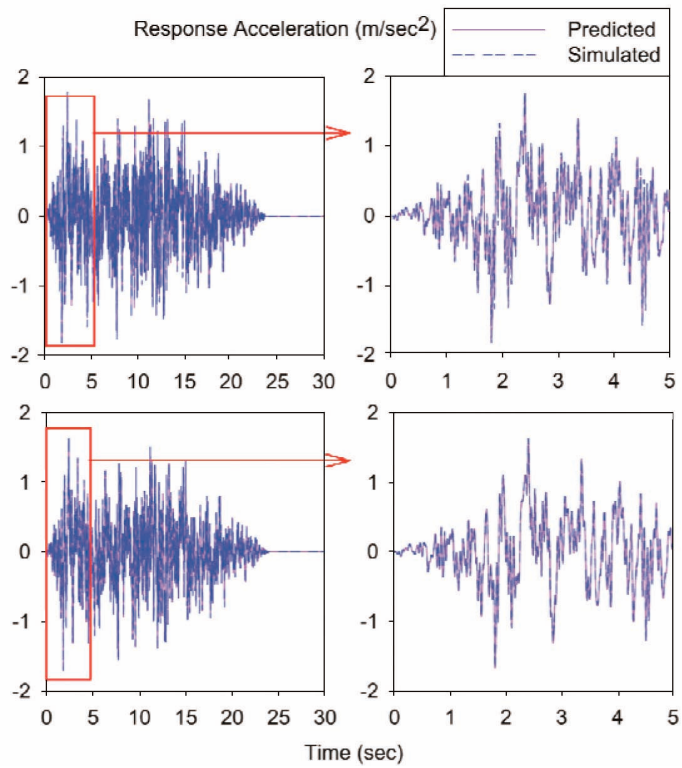

Fig. 12. Comparison of Seismic Acceleration Responses for the Frame Model
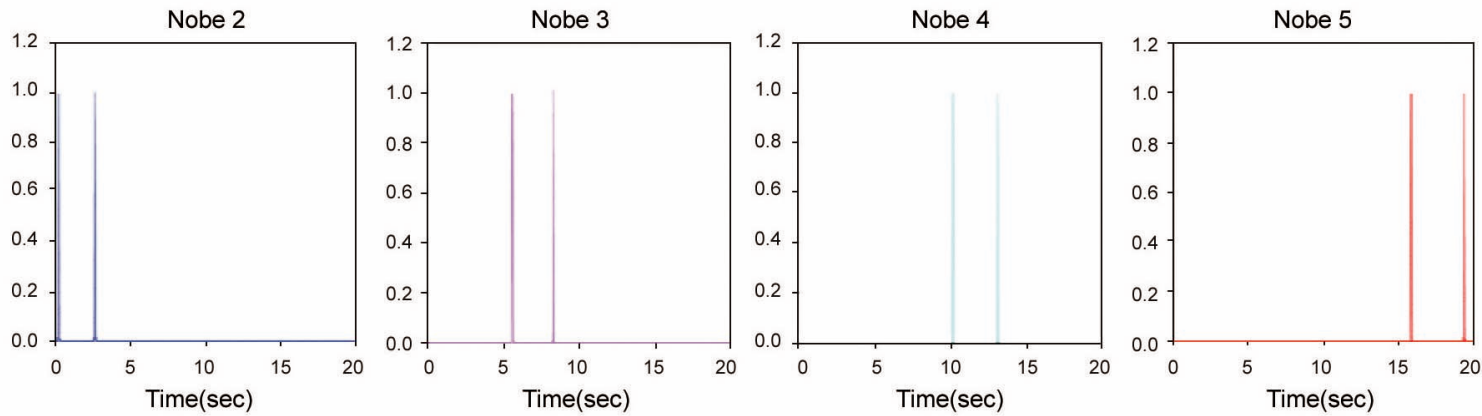

Fig. 10. Impact Forces for the Frame Model
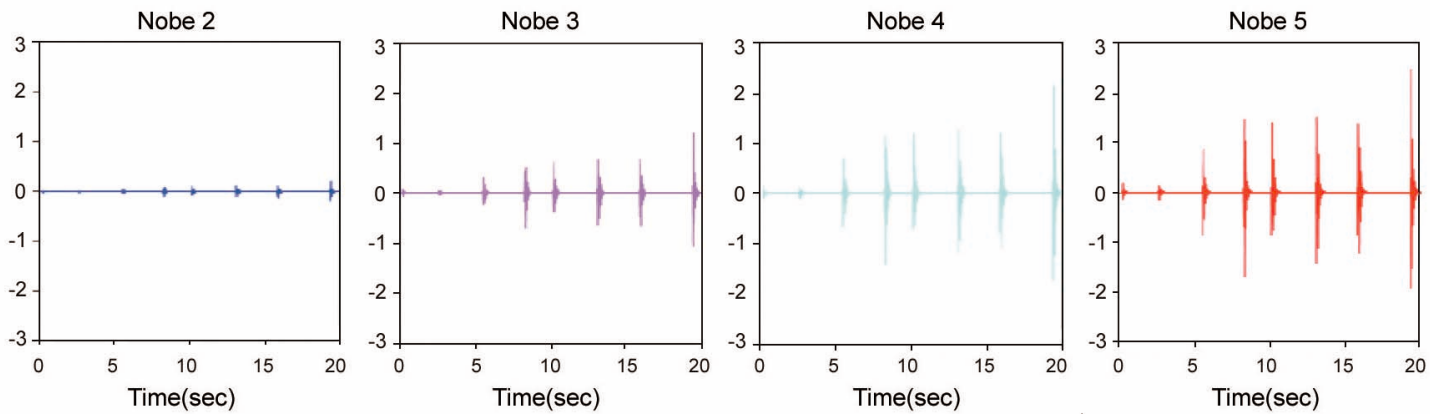

Fig. 11. Floor Acceleration Responses for the Frame Model 

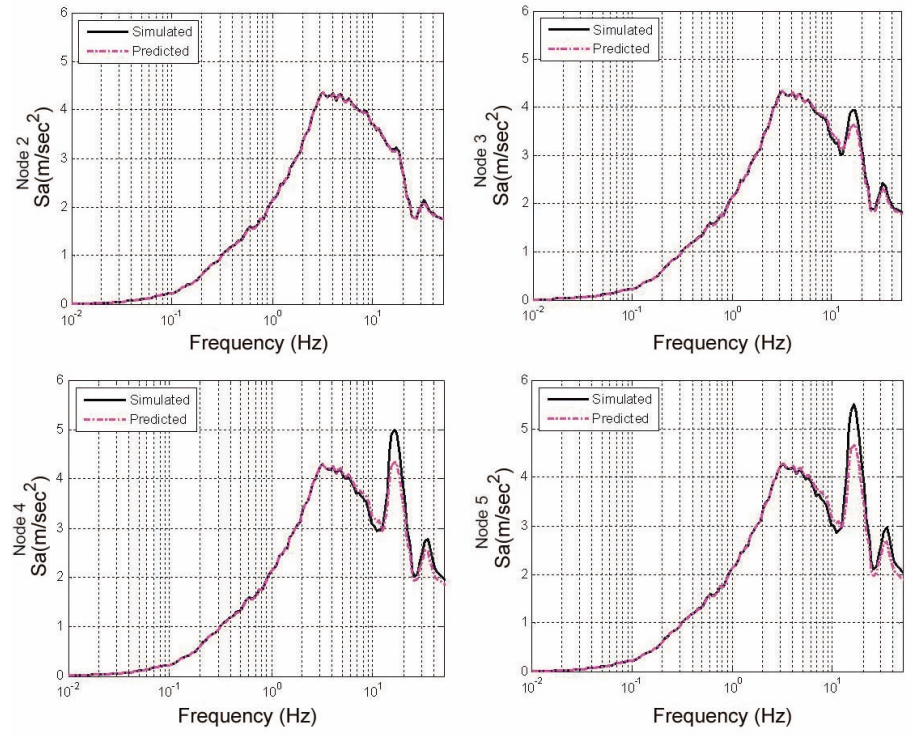

Fig. 13. Comparison of Simulated and Predicted ICRS's for the Frame Model
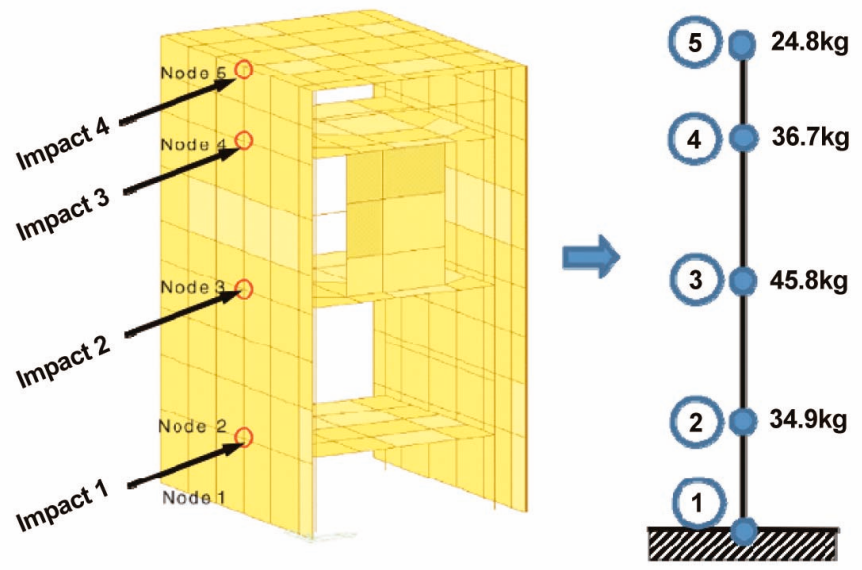

Fig. 14. Idealization of the 3-D Finite Element Model
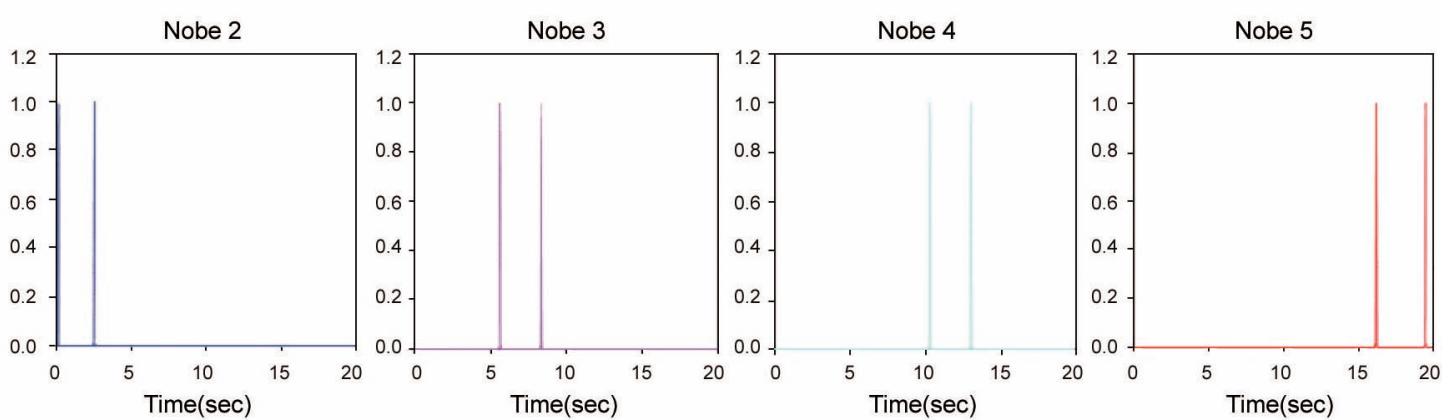

Fig. 15. Impact Forces for the 3-D Finite Element Model 

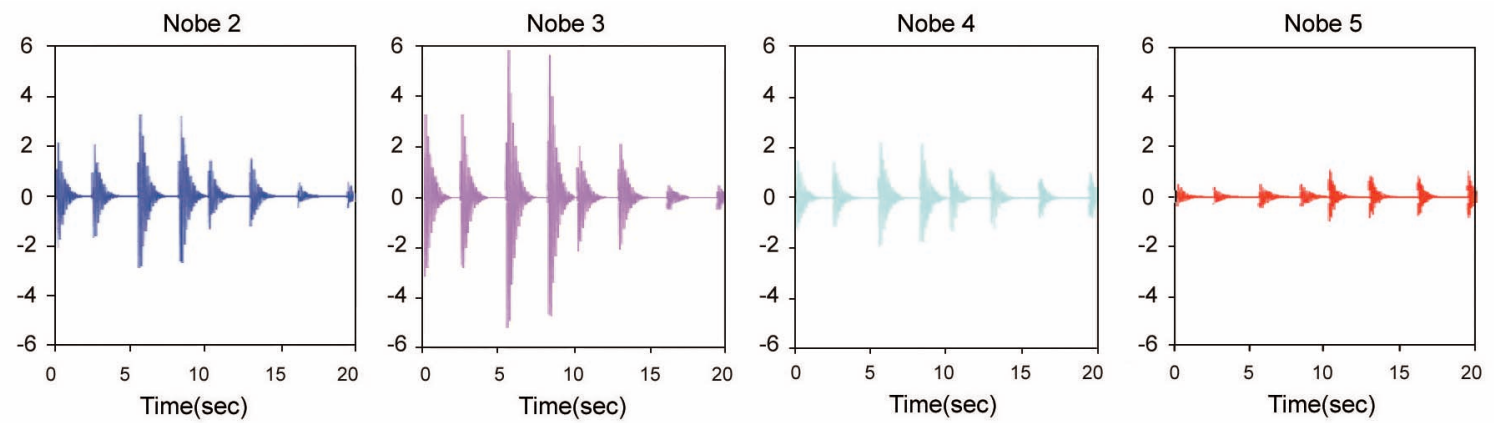

Fig. 16. Floor Acceleration Responses for the 3-D Finite Element Model
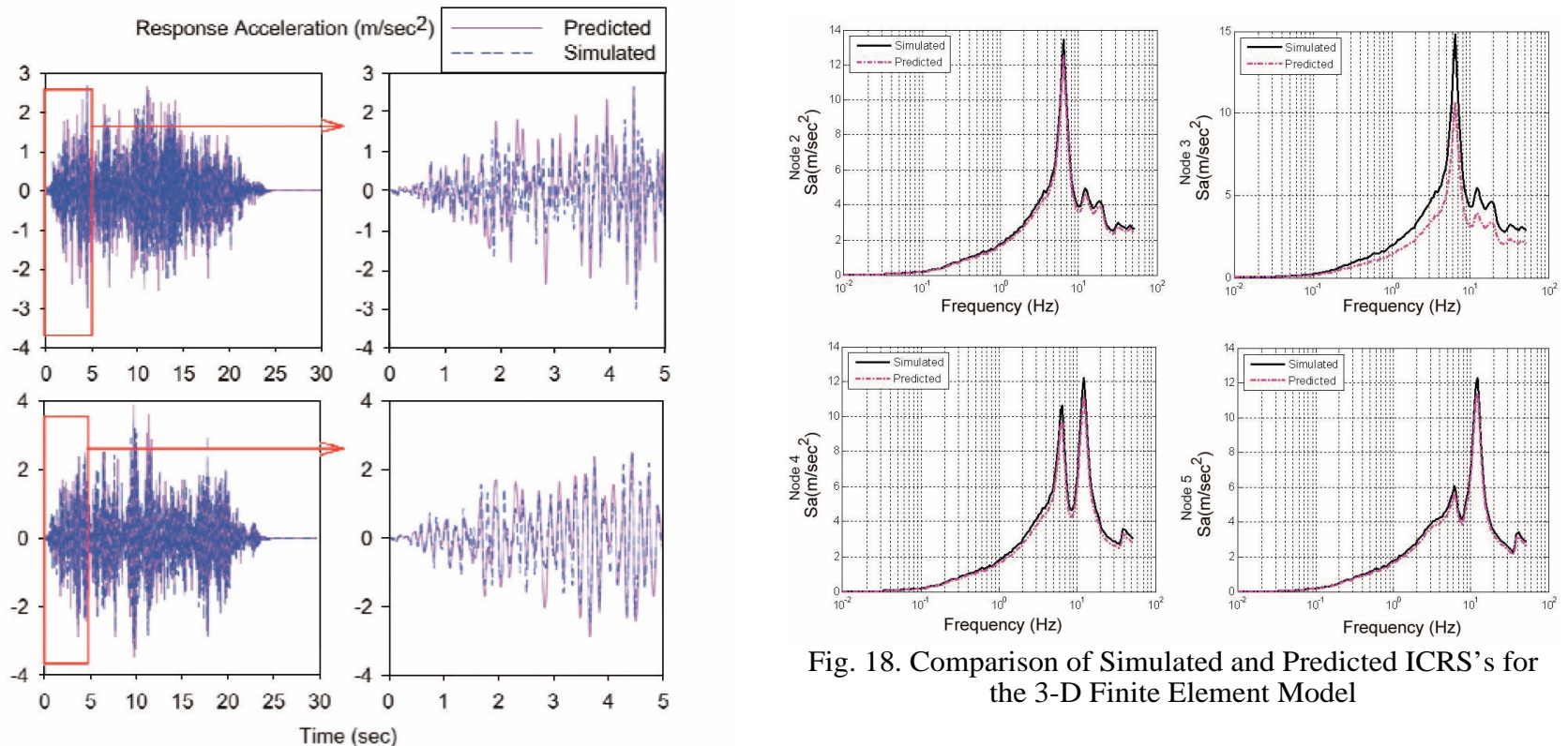

Fig. 18. Comparison of Simulated and Predicted ICRS's for the 3-D Finite Element Model

Fig. 17. Comparison of Seismic Acceleration Responses for the 3-D Finite Element Model

and predicted earthquake responses at nodes 3 and 4 are compared in Fig. 17. The predicted response shows reasonable agreement with the measured response even with some differences in the time variation. The comparison of predicted and simulated mean ICRS shows good matches as shown in Fig. 18. The slight difference in the peak spectral acceleration resulted from the nodal mass estimation error and the simplification of the model.

\section{CONCLUSION AND DISCUSSION}

The ICRS prediction procedure is presented and verified by numerical simulation tests using three structural models. From comparisons of the predicted and simulated ICRS

of the numerical models, the proposed algorithm appears to efficiently predict the ICRS of the cabinet structures.

The presented procedure eliminates uncertainty associated with constructing the analytical model of an electrical cabinet having complex mass distribution and stiffness. The proposed method is especially effective and efficient for cabinet structures now operating in nuclear power plants. It can be performed reliably with the aid of impact hammer tests, but it is not suitable for shaking table tests, as cabinets already installed in the operating plants cannot be removed from the plants to be mounted on a shaking table. The proposed algorithm can be developed as a candidate algorithm for ICRS prediction of cabinet structures. It is expected that the procedure proposed in this study will be used in the SQ of electrical equipment installed in operating plants.

For further study, experimental validations might be 
performed to obtain more confidence in the proposed algorithm. There is also a possible problem with obtaining the lumped mass matrix since the exact weights of a cabinet system may not be readily available. It may be necessary to develop a way to estimate the lumped mass matrix from impact hammer tests.

\section{ACKNOWLEDGEMENTS}

This work was supported by the Nuclear Innovation Program of the Korea Institute of Energy Technology Evaluation and Planning (KETEP) grant funded by the Korea Ministry of Knowledge Economy (No. 201016201 00020). The authors would like to express their appreciation for the financial support.

\section{REFERENCES}

[ 1] IEEE Power Engineering, IEEE Recommended Practices for Seismic Qualification of Class 1 E Equipment for Nuclear Power Generating Stations, IEEE Std 344-2004, The Institute of Electrical and Electronics Engineers Inc., 2005.

[2] O'sullivan, J. J. and Djordjevic, W., Guidelines for Development of In-Cabinet Amplified Response Spectra for Electrical Benchboards and Panels, EPRI NP-7146M, Electric Power Research Institute, Palo alto, CA, 1990.

[3 ] Gupta, A., Rustogi, S. K., and Gupta, A. K., "Ritz vector approach for evaluating in-cabinet response spectra," Journal of Nuclear Engineering and Design, 190, 255272, 1999.

[4] Gupta, A., and Yang, J., "Modified Ritz vector approach for dynamic properties of electrical cabinets and control panels," Journal of Nuclear Engineering and Design, 217, 49-62, 2002.

[ 5 ] Yang, J., and Gupta, A., "INCABS: A Computer Program for Evaluating Incabinet Spectra," Transactions of SMiRT 16, Paper \#2072, Washington DC, August 2001.

[6] Rustogi, S., and Gupta, A., "Modeling the Dynamic Behavior of Electrical Cabinets and Control Panels: Experimental and Analytical Results," Journal of Structural Engineering, 130, No. 3, 511-519, 2004.

[7] Koo, K. Y., Cho, S. G., Cui, J. and Kim, D., "Seismic response prediction for cabinets of nuclear power plants by using impact hammer test," Journal of Nuclear Engineering and Design 240, 2500-2511, 2010. DOI: 10.1016 /j.nucengdes.2010.05.008.

[ 8 ] Soong, T. T., Active Structural Control: Theory and Practice, John Wiley \& Sons, Inc., New York, 1990.

[9] Overschee, P. van, and Moor, B. de, "N4SID: Subspace algorithms for the identification of combined deterministicstochastic systems," Automatica, 30, 75-93, 1994.

[10] Overschee, P. van, and Moor, B. de, 1997, "Closed-loop subspace system identification," Proc. of the 36th Conference on Decision and Control, pp. 1848-1853, 1997.

[11] Kailath, T., Linear Systems, Prentice Hall, Englewood Cliffs, NJ, USA, 1980.

[12] Ljung, L., System Identification: Theory for the User, 2nd Edition, Prentice-Hall, NJ 07458, USA, 1999.

[13] Overschee, P. van, and Moor, B. de, Subspace Identification for Linear Systems, Kluwer Academic Publishers, Boston, MA, 1996.

[14] Overschee, P. van, and Moor, B. de, "A Unifying Theorem for Three Subspace System Identification Algorithms," Automatica, 31, 1853-1864, 1995.

[15] MIDAS Civil 2006 Online Manual, MIDAS Information Technology Co., Ltd., Seoul, 2005.

[16] Joe, Y. H., Park, H. G., and Cho, S. G., "Comparative Study of Analytical Modal Properties of Instrumentation Cabinet of Nuclear Power Plant," Proceedings of EESK Conference-Fall 1999, 3, Incheon, Korea, September 18, 1999 (in Korean). 